East African Medical Journal Vol. 81 No. 7 July 2004

MORBIDITY AND MORTALITY OF LOW BIRTH WEIGHT INFANTS IN THE NEW BORN UNIT OF KENYATTA NATIONAL HOSPITAL, NAIROBI D. E. Simiyu, MBChB, MMed, (Paediatrics) Cert. Neonatology (Tel Aviv), Lecturer, Department of Paediatrics and Child Health, Faculty of Medicine, College of Health Sciences, University of Nairobi, P.O. Box 19676, Nairobi, Kenya

\title{
MORBIDITY AND MORTALITY OF LOW BIRTH WEIGHT INFANTS IN THE NEW BORN UNIT OF KENYATTA NATIONAL HOSPITAL, NAIROBI
}

\author{
D. E. SIMIYU
}

\begin{abstract}
Background: Morbidity and mortality of low birth weight (LBW) infants at Kenyatta National Hospital (KNH) has previously been found to be high. Other centres have shown that even with lack of neonatal intensive care facilities, selective interventions can be implemented that improve neonatal survival rates. It is important to identify those factors at KNH that when selectively modified, will improve the quality of care hence survival rates.

Objective: To quantify the morbidity and mortality of LBW infants in KNH. To audit the quality of care and identify factors that can be selectively modified to improve the quality of care and improve the currently low survival rates.

Design: Retrospective study utilising case notes.

Setting: New born unit, Kenyatta National Hospital, Nairobi.

Subjects: All LBW infants admitted to the NBU at KNH from January to December 2000. Results: Out of an expected 694 files, 533 (77\%) were studied. The male to female ratio and LBW to VLBW ratio was 1:1 respectively. Small for gestational age (SGA) accounted for $\mathbf{1 1 . 6 \%}$. Overall mortality was $\mathbf{5 7 . 4 \%}$ (574/1000 admissions) while mortality for SGA was $37 \%$. Infants born out of $\mathrm{KNH}$ had significantly higher mortality (p=0.0047). Compared to Caeserian delivery, infants born via spontaneous vertex delivery had higher mortality $(\mathrm{p}=\mathbf{0 . 0 0 8 7})$. The leading diagnoses on admission or death were respiratory distress $(69 \%)$, apnoeic attacks (42\%) suspected sepsis and jaundice (37\% each), hypothermia $(27 \%)$ and anaemia $(17 \%)$. By time of death or discharge, $43 \%$ had no laboratory investigations done. While $37 \%$ had suspected sepsis, only $14 \%$ had blood culture done. Antibiotics were started in 460 (86\%) of infants yet only $37 \%$ had diagnosis of suspected sepsis. Change of antibiotics was guided by culture and sensitivity reports in only 62(13.5\%). Apnoeic spells were managed with rectal aminophyline in 156(29\%) infants of whom 19(12\%) survived. The terminal events for the dead infants included recurrent apnoeic spells. The only mode of nutrition was enteric feeding in $59 \%$ with breast milk, formula or pasteurized cows milk by nasogastric tube, breast feeding or cup feeding. Except for dextrose water, parenteral nutrition was not utilised. Of 63 infants dying with associated anaemia, $43(68 \%)$ were not transfused due to lack of reliable blood supply.

Conclusion: Compared to previous studies, mortality of LBW infants in KNH- NBU has increased over the years. Improvement in supportive care as regards nutrition, temperature regulation, laboratory back up and respiratory support is recommended. There is a place for trial of kangaroo mother care and simple continous positive airways pressure administration methods. There is a need to ascertain the efficacy of the method of aminophyline administration.
\end{abstract}

\section{INTRODUCTION}

Mortality and morbidity of low birth weight infants is related to their birthweight and gestational age(1). Quality of care has significant influence on the survival of these infants. In the industrialized countries, continued improvement in the quality of care has resulted in improvement in survival rates for these infants. In contrast, the developing countries while shouldering $90 \%$ of the world's burden of low birth weight infants only has $10 \%$ of the world's resources for their care. The survival rates for these infants has therefore remained comparatively low.

Low birth weight (LBW) related to intrauterine growth retardation (IUGR) is known to carry high morbidity and mortality especially at term(2). In the developing countries, it has been suggested that a substantial number of LBW infants also have IUGR(3). This may have a negative impact on the survival rates of LBW infants in this countries. 
The neonatal survival rate for LBW infants at the $\mathrm{NBU} / \mathrm{KNH}$ is still much lower than that observed in other centres of the developing world where there are no neonatal intensive care facilities. This survival rate is also lower than that reported from developed countries prior to the advent of neonatal intensive care services(4). This may imply a deficiency in our neonatal services that has resulted in this high mortality. Implementation of neonatal intensive care services alone may not be adequate to improve our low birth weight survival rates.

In the mid eighties, Adekunle and Effiong in Nigeria while working in a neonatal intensive care deficient unit, recorded an improvement in survival of neonates after instituting selective interventions in the course of their care(5).

It is therefore necessary to identify the causes of low survival rates at $\mathrm{KNH}$ and suggest interventions based on the Adekunle and Effiong model that will improve these rates even before the advent of neonatal intensive care services in this hospital.

\section{MATERIALS AND METHODS}

The study was conducted at the records department of KNH. All records of newborns admitted to the NBU from January to December 2000 were accessed from the departments data base. From these, all with LBW were identified by registration number. Each file of the LBW was then traced and retrieved manually. The reseacher scanned the files and extracted the relevant information. The files were for both the deceased and those discharged alive from the NBU. The neonatal period was defined as that period from birth upto the age of twenty eight days while LBW was defined as birth weight less than 2,500 grams. A coded data sheet was prepared and used to extract the following information.
Maternal/ Obstetric data: Mothers age, marital status, gestation age by dates, place of delivery, mode of delivery and indications if applicable, Apgar score (recorded or inferred from history) and any maternal illnesses.

Infant data: Sex, maturity (by dates and clinical assessment), birth weight, diagnosis, investigations done, therapeutic interventions, nutrition, duration of stay, outcome (dead or alive upto discharge).

Ethical considerations: The study was approved by the ethics and research committee of KNH. Confidentiality was ensured by using inpatient registration numbers rather than patients names.

Data management: The data was entered in a computer and analysed on the SPSS programme (statistical package for social studies). Rates, ratios, percentages were calculated. Where applicable, measures of significance were derived by Chi-square and Fisher exact tests. This data was presented in tables where appropriate.

\section{RESULTS}

A total of 533 records out of the expected 694 were traced and retrieved (77\%). Some records lacked information on different aspects such as maternal age $82(15.4 \%)$, gestational age $104(19.5 \%)$ and marital status $83(15.5 \%)$. A total of $451(84.6 \%)$ of the records had maternal age stated. The age ranged from 13 years to 43 years with a median of 23 years. Adolescent mothers were $79(17.5 \%)$ while those aged over 35 years were $19(4.2 \%)$. The majority of mothers, $280(52.5 \%)$ were married while for $83(15.5 \%)$ the marital status was not indicated. The remaining $171(32 \%)$ were single. Of those who were single, 56(33\%) were adolescents. Majority of mothers attended antenatal clinic $307(68 \%)$ at least twice in the course of the pregnancy. Adolescent mothers were less likely to attend antenatal clinic (Table 1).

Table 1

Antenatal clinic attendance and maternal age

\begin{tabular}{lccc}
\hline Maternal age (years) & ANC & No ANC & Total \\
& No. $(\%)$ & No. $(\%)$ & No. $(\%)$ \\
\hline$<20$ & $43(54.4)$ & $36(45.6)$ & $79(17.5)$ \\
$20-35$ & $249(70.5)$ & $104(29.5)$ & $353(78.3)$ \\
$>35$ & $15(79)$ & $4(21)$ & $19(4.2)$ \\
\hline Total & $307(68)$ & $144(32)$ & $451(100)$ \\
\hline
\end{tabular}

Chi square $=7.65, \mathrm{P}=0.006$

A significant number of those referred from other hospitals or born out of hospital died. For six infants, the place of delivery was not recorded. The mode of delivery was not specified for $16(3 \%)$. Mortality was higher for spontaneous vertex delivery $(62.3 \%)$ and breech extraction $(63.3 \%)$ compared to caeserian section $(45.2 \%)$. This difference was significant for spontaneous vertex delivery $(\mathrm{P}<0.05)$ but not for breech extraction $(\mathrm{P}=0.074)$ when compared with caeserian delivery (Table 2). 
Table 2

Mortality in relation to place and mode of delivery

\begin{tabular}{|c|c|c|c|c|}
\hline Maternal variable & $\begin{array}{c}\text { Alive } \\
\text { No. }(\%)\end{array}$ & $\begin{array}{c}\text { Dead } \\
\text { No. }(\%)\end{array}$ & Chi-Square & P-value \\
\hline \multicolumn{5}{|l|}{ Place of delivery } \\
\hline Hospital & $189(45)$ & $231(55)$ & 7.98 & 0.0047 \\
\hline Non-hospital & $32(30)$ & $75(70)$ & & \\
\hline \multicolumn{5}{|l|}{ Hospital of delivery } \\
\hline $\mathrm{KNH}$ & $167(47)$ & $188(53)$ & 3.87 & 0.049 \\
\hline Other hospitals & $22(33.8)$ & $43(66.2)$ & & \\
\hline \multicolumn{5}{|l|}{ Mode of delivery } \\
\hline SVD & $137(37.7)$ & $226(62.3)$ & 11.08 & 0.0087 \\
\hline $\mathrm{C} / \mathrm{S}$ & $68(54.8)$ & $56(45.2)$ & & \\
\hline Breech extraction & $11(36.7)$ & $19(63.3)$ & 3.19 & 0.074 \\
\hline
\end{tabular}

$\mathrm{KNH}=$ Kenyatta National Hospital, $\mathrm{SVD}=$ Spontaneous delivery, $\mathrm{C} / \mathrm{S}=$ Caeserian secretion.

Mortality was highest among infants of older mothers (>35 years) followed by adolescents. However the difference was not significant (Table 3)

Table 3

Maternal age versus infant mortality

\begin{tabular}{|c|c|c|c|c|c|c|c|c|c|}
\hline \multirow{2}{*}{ Variable } & \multicolumn{5}{|c|}{ Maternal age group (years) } & & \multirow{3}{*}{$\begin{array}{l}\text { Odds } \\
\text { ratio* }\end{array}$} & \multirow{3}{*}{$\begin{array}{c}95 \% \\
\text { CI* }\end{array}$} & \multirow[t]{3}{*}{$\mathrm{P}$ value* } \\
\hline & $\begin{array}{r}<20 \\
(\mathrm{n}\end{array}$ & & $\begin{array}{c}20-35 \\
(n=353)\end{array}$ & & & & & & \\
\hline Mortality & No. & $(\%)$ & No. & $(\%)$ & No. & $(\%)$ & & & \\
\hline Alive31 & 39.4 & 153 & 43 & 7 & 37 & & \multirow[b]{2}{*}{0.76} & & \multirow[b]{2}{*}{0.570} \\
\hline Dead & 48 & 61 & 200 & 57 & 12 & 63 & & $\begin{array}{l}0.47 \\
1.55\end{array}$ & \\
\hline
\end{tabular}

$\mathrm{CI}=$ Confidence interval $*=$ This is comparison of adolescents to the age group $20-35$ years

Prolonged rupture of membranes (PROM) and preeclampsia/eclampsia complicated $65(11.3 \%)$ and 47 $(10.4 \%)$ of the deliveries respectively. Pre-eclampsia/ eclampsia rates were similar across the maternal age spectrum while PROM was more frequent in the mothers aged over 35 years (15.8\%).

Infants characteristics: Out of the 533, there were $276(51.8 \%)$ males and 257(48.2\%) females making a M: $\mathrm{F}$ ratio of approximately 1:1. There were 306 deaths making a mortality rate of $57.4 \%$ or $574 / 1000$ admissions. There was a slight male preponderance among the dead but this was not statistically significant (Table 4). There was equal distribution between VLBW and LBW 269 and 264 respectively. VLBW infants had significantly higher mortality than LBW infants $(\mathrm{p}=0.000)$. Gestational age was not recorded for $100(18.8 \%)$ of the infants. Gestational age $<31$ weeks had significantly higher mortality than those $>31$ weeks.(Table 4 ).

Table 4

Mortality in relation to infant variables

\begin{tabular}{|c|c|c|c|c|c|}
\hline Infants variable & & $\begin{array}{l}\text { Alive } \\
\text { No. } \%\end{array}$ & $\begin{array}{r}\text { Dead } \\
\text { No. \% }\end{array}$ & Chi-square & P-value \\
\hline \multicolumn{6}{|l|}{ Sex } \\
\hline & Male & $113(41 \%)$ & $163(59)$ & 0.65 & 0.42 \\
\hline & Female & $114(44.4)$ & $143(55.6)$ & & \\
\hline \multicolumn{6}{|l|}{ Birth weight } \\
\hline & $\leq 1500 \mathrm{~g}(\mathrm{VLWB})$ & $44(16.4)$ & $225(83.6)$ & 152.85 & $\mathrm{P}=0.000$ \\
\hline & $1501-2499$ & $183(69.3)$ & 81 (30.7) & & \\
\hline \multirow[t]{2}{*}{ Gestation } & $\leq 31$ & $24(15)$ & $135(85)$ & 95.01 & $\mathrm{P}=0.000$ \\
\hline & $>31$ & $174(63.5)$ & $100(36.5)$ & & \\
\hline
\end{tabular}


The diagnosis of small for gestational age or IUGR could not be ascertained because most of the recorded gestation $(70 \%)$ was based on dates and not clinical assessment. However from this records, 62(11.6\%) were small for gestational age and of these, 23(37\%) died reflecting a mortality rate lower than the overall rate of $57.4 \%$.

Average duration of stay was 10.2 days but for those who lived to discharge, the average duration of stay was 18.7 days. For those who died, 111(36\%) died within 24 hours of admission and a further 62 (20\%) died within the first seven days of admission. (Cumulative death in first week was $56.5 \%$ ).
The leading clinical diagnoses on admission or death were respiratory distress, apnoeic attacks, suspected sepsis, jaundice and hypothermia. Meningitis was only considered in eight infants. Infants had more than one morbidity event at admission or death hence the total in the columns is not equal to 533. All morbidity events were associated with mortality rate greater than $40 \%$ except confirmed sepsis and SGA.

Some events such as apnoeic attacks, hypothermia and birth asphyxia were associated with mortality greater than $80 \%$. Similarly, convulsions, dehydration and suspected sepsis were associated with mortality greater than $70 \%$ (Table 5).

Table 5

Morbidity events and subsequent mortality

\begin{tabular}{lccr}
\hline Morbidity event & Alive & Dead & Total \\
& No. $(\%)$ & No. $(\%)$ & No. $(\%)$ \\
\hline Respiratory distress & $123(33.5)$ & $244(66.5)$ & $367(69)$ \\
Apnoeic attacks & $7(3)$ & $152(76.8)$ & $226(42.4)$ \\
Suspected sepsis & $46(23.2)$ & $121(61.1)$ & $198(37.1)$ \\
Jaundice & $77(38.9)$ & $123(84.8)$ & $198(37.1)$ \\
Hypothermia & $22(15.2)$ & $63(69.2)$ & $145(27.2)$ \\
Anaemia & $28(30.8)$ & $55(705)$ & $78(17)$ \\
Dehydration & $23(29.5)$ & $27(36.5)$ & $74(13.9)$ \\
Confirmed sepsis & $47(63.5)$ & $23(37)$ & $161(11.6)$ \\
SGA & $39(63)$ & $50(82)$ & $61(11.4)$ \\
Birth asphyxia & $11(18)$ & &
\end{tabular}

$\mathrm{SGA}=$ Small for Gestational Age

Those with confirmed sepsis had the longest average duration of stay of 20.3 days. No positive urine or cerebral spinal fluid cultures were found and sepsis was diagonised on blood culture. The common bacteria were gram negative organisms (66.6\%) namely klebsiella, citrobacter, enterobacter and pseudomonas in that order. Gram positive bacteria accounted for the rest and this were coagulase negative staphylococci, enterococci and alkaligens fecalis. One case of lancefield group B Beta haemolytic streptococci was found.

Quality of care: Investigations: A total of 230 infants (43\%) were not investigated at all, six (37.4\%) were discharged without any investigation while 144 $(62.6 \%)$ died before any investigations were carried out. For the 303(57\%) that were investigated, a total of 1,173 venepunctures were undertaken averaging four venepunctures per infant. Majority venepunctures were for bilirubin estimation 347(29.6\%), 316(27\%) were for haemogram, $163(13.9 \%)$ were for blood culture. The remaining $29.5 \%$ of venepuncutres were shared between blood grouping, urea and electrolytes estimation, HIV serology, VDRL, hepatitis B surface antigen and serum proteins.

Lumber puncture for cerebrospinal fluid was carried out in only four of the eight clinically thought to have meningitis. Despite the high number of children with respiratory distress and apnoeic attacks, only 17 blood gas analysis were done. Many investigations as reported were indicated in the file as carried out but the reports from the laboratory were missing. As an example, of the 163 blood cultures done, 57 (35\%) of the reports were missing from the files (Table 6).

Table 6

Frequency of investigations

\begin{tabular}{lccc}
\hline Investigation & Alive & Dead & Total \\
\hline No investigation & 86 & 144 & 230 \\
Bilirubin & 204 & 143 & 347 \\
Haemogram & 185 & 131 & 316 \\
Blood culture & 97 & 66 & 163 \\
Blood group & 54 & 32 & 86 \\
UREA electrolytes & 14 & 23 & 37 \\
estimation & & & \\
Chest X-Ray & 6 & 20 & 26 \\
Blood sugar & 7 & 16 & 23 \\
Blood gases & 1 & 16 & 17 \\
HIV & 12 & 3 & 15 \\
\end{tabular}

Antibiotic use: Out of the 533 infants admitted, $460(86 \%)$ were started on antibiotics yet only 198 $(37 \%)$ had clinical diagnosis of suspected septicaemia. 
The average duration of antibiotic use was seven days. For the 227 who survived to discharge, 173(76\%) were on antibiotics. The commonly used combination being crystalline penicillin and gentamicin. Of those on antibiotics, 73(42\%) had the antibiotics changed to mainly a 3rd generation cephalosporin and aminoglycoside. This change was guided by culture and sensitivity reports in $35(48 \%)$.

For the 306 who died, 287(94\%) were on antibiotics again commonly crystalline penicillin and gentamicin. The 19 that were not on antibiotics died immediately on admission. Antibiotic regimen was changed in $123(43 \%)$ but only $27(22 \%)$ were guided by culture and sensitivity reports.

Theophylline/Aminophylline therapy: Aminophylline is routinely administered rectally to premature infants weighing less than $1500 \mathrm{~g}$ who have respiratory distress as a policy in the NBU. There is however no facility for monitoring the subsequent theophylline serum levels and no studies have been done to ascertain the efficacy of this method of administration. In this study, 156 (29\%) of LBW infants were on aminophylline rectally at a dose of $2 \mathrm{mg} / \mathrm{kg}$ body weight in two divided doses in 24 hours. Of those on aminophylline only 19(12\%) were alive to discharge.

Intravenous fluid therapy/ Fluid electrolyte balance: Intravenous access was universally via a peripheral vein utilizing gauge 22 to 23 catheters. Most infants 475 $(89.1 \%)$ were started on intravenous fluids. The average duration of intravenous fluid therapy was 3.2 days. The initial intravenous fluid used was $10 \%$ dextrose water in $428(90 \%)$ of cases. Five percent dextrose water in $29(6 \%)$ and the rest $18(4 \%)$ were on half strength darrows, normal saline or the type was not indicated. Addition of potassium chloride and normal saline to the infusate was routinely carried out after the initial 24 hours of intravenous fluids.

Nutrition: No infant received parenteral nutrition other than dextrose water. The mode of nutrition was enteral utilizing nasogastric tube, cup feeding or breastfeeding.

A total of 342 infants were fed enterally with 314 $(92 \%)$ of these tolerating their feeds. Breast milk was utilized in 244(77.7\%), formula in 39(12.3\%) and pasteurized cows milk in $31(10 \%)$. The reason for using formula or cows milk (when this were indicated) were, maternal death, mother absconded, ill health after caeserian section or HIV positive status.

Blood transfusion: A total of $91(17 \%)$ were diagnosed to have anaemia both clinically and by haemoglobin estimate. Forty eight $(52.7 \%)$ of these infants were transfused with eight being transfused twice and two being transfused thrice. For those infants diagnosed with anaemia and survived, all were transfused while of those diagnosed with anaemia and died, only $20(31.7 \%)$ had been transfused (Table 4$)$. The reasons for not transfusing was unavailability of compatible blood from the blood bank. Four infants died while on transfusion while one died for lack of veinous access.
Exchange transfusion: A total of six exchange transfusions out of an intended nine were performed. Three of those undergoing the procedure died subsequent to the procedure. The cause of death was not related to the procedure. For the three who were scheduled for the procedure but did not receive it, all died prior to the procedure with the reason for delaying the procedure being lack of compatible blood.

Phototherapy: All 198 infants diagnosed with jaundice underwent phototherapy. For those who lived to discharge, the average duration of phototherapy was five days while for those who died, this was three days.

\section{DISCUSSION}

The retrieved records formed $77 \%$ of those actually admitted with diagnosis of LBW. Those missing records were for both those who survived to discharge and those who died.

Several studies have previously been done on morbidity and mortality of neonates in the $\operatorname{NBU}(4,6,7)$ but only one has specifically studied the morbidity and mortality of low birth weight infants and even this one cut off at birth weight 1,999 grammes hence leaving out the weight category of 2000 - 2499 grammes(4). None of these studies looked at the management practices in the unit that had an impact on the survival or otherwise of LBW infants. Our study attempts to resolve the issue so as to determine the significant contributors to morbidity and mortality of LBW infants in terms of David and Siegels terminology of "better babies or better care"(8).

Maternal characteristics: In our study, majority of mothers were in the age group 20-35 years. Various studies have found that extremes of maternal age was associated with increased morbidity and mortality of their infants(9-13). In our study, the adolescent mothers were less likely to have attended antenatal care. This may have contributed to the higher mortality of their offspring $(14,15)$. Antenatal care attendance was shown by Elster to have positive influence on the outcome of adolescent pregnancy. In our study adolescent mothers were likely to be single. However marital status is a doubtful contributor to LBW morbidity and mortality $(16,17)$. Zuckerman et al. showed that it is the unfavourable socio economic status of the adolescent that confers increased neonatal morbidity and mortality rather than the adolescent state per se(15).

Infants born out of the admitting hospital have previously been reported to suffer higher morbidity and mortality $(6,7,18)$ while other workers failed to confirm this(19,20). In our study, out-born infants suffered significantly higher mortality than in-born infants. This could be a reflection of poor transfer facilities between hospitals or adverse perinatal events surrounding the particular deliveries.

Caeserian section has previously been advocated as the method of choice for delivery of LBW infants. 
In our study, the mortality was higher for vaginally delivered infants compared to caeserian section. Jacob and co-workers in Alaska found this to be the case for the Alaskan natives(20) while Melchor and co-workers found no advantage of caeserian section but they looked specifically at very low birth weight infants $(<1500 \mathrm{~g})(21)$.

Infants characteristics: In our study, the male to female ratio was approximately $1: 1$. This agrees with Kramer's 1987 meta analysis which found no overall difference in sex distribution among LBW infants(17).

The mortality rate for LBW infants of 574/1,000 admissions found in our study is more than double the rate $(270 / 1,000)$ reported by Meme from the same hospital in 1978 (6). It is also substantially higher than the rate reported by Kasirye and Musoke $(384 / 1,000)$ in 1992(7). This 1992 rate is almost similar to that reported by Were, Mukhwana and Musoke (374/1000 by inference from study results) in 2002 for a study done in 1996(4). This latter rate of 1996 left out one weight category of 2000-2499 grammes. This apparent increase of LBW mortality between 1978 and 2000 can be explained by various factors.

(a) The missing records that totaled 161(23\%) if all were assumed to be records of survivors then the mortality rate reduces to 440/1,000 - but even this represents over $63 \%$ increase in mortality between 1978 and 2000 .

(b) Due to the small sample size of the 1996 study and the fact that one weight category (2000-2499 grammes) was not included in that study, the increasing rate may have been missed.

(c) Increased high risk deliveries at $\mathrm{KNH}$ over the years and referral of LBW infants from both city health facilities and surrounding rural hospitals. The number of deliveries at $\mathrm{KNH}$ have actually doubled from about 3,600 annually in 1978 to about 7,500 in the year 2000 (22). This has resulted in overcrowding, understaffing, breakdown in infection control measures and poor work environment.

(d) The enforcement of world bank/IMF sponsored programmes has reduced funding for health care hence equipment maintenance, replacement and new acquisition has been compromised.

The use of birth weight and gestational age to predict neonatal mortality was advocated by Lubchenco and co-workers as a method to identify infants at risk and intervene appropriately(1). From our study, mortality appears to have worsened from 1978 to 2000 . Identifying those at increased risk by birth weight and gestation age does not appear to have improved their survival.

LBW infants who live to discharge are also known to suffer higher post neonatal mortality(23). If this is so for the graduates of the KNH- NBU then fewer infants ultimately survive than what we are reporting.

Those dying within 24 hours in our study are a higher percentage $(36 \%)$ than that reported by Were in 1996 (28\%). However commulative mortality within the first week found in this study, $(56.5 \%)$ is lower than that found by Were, (70\%) (4). Kasirye's study was not restricted to LBW infants alone, his rate for the 1 st week was higher at $86.8 \%$ in $1992(7)$. It is difficult to say whether this apparent reduction in mortality in the week found in this study is a reflection of better care in the first week only for the infants to succumb later. This difference cannot be attributed to "better babies" because perinatal asphyxia was still a prominent morbidity event in all these studies from 1978 to 2000.

Compared to previous studies, apnoeic attacks, hypothermia and dehydration are prominent morbidity events in our study. The previous studies being prospective may have recorded different rates of various morbidities because in a research situation, patient care tends to somewhat improve as the researcher is more vigilant. There is no report of dehydration from Kasirye's nor Were's reports, hypothermia is not mentioned by Were while Kasirye mentions it together with apnoe as morbidity events in the LBW infants in his study but not contributing to their mortality $(4,7)$. In a recent report from the Ethio-Swedish childrens hospital in Addis Ababa Ethiopia, hypothermia is reported as affecting $90 \%$ of LBW infants(24). This is higher than the rate we are reporting.

In our study, apnoeic attacks were prominent terminal events. In over $70 \%$ of the infants who died, associated morbidity events included hypothermia, asphyxia, hypoglycaemia, necrotising enterocolitis, convulsions, suspected sepsis, congenital anomalies and dehydration in that order. Anaemia, jaundice and intra uterine growth retardation are also important morbidity events in this study but together only contributed to $8.74 \%$ of the clinical syndromes in Were's study(4). The reason for the above differences in reported morbidity events could be because unlike the other studies, this study aimed at identifying these events and subsequent clinical practices that could be improved to enhance survival. Respiratory failure (apnoeic attacks), poor temperature control (hypothermia) lack of supportive care (dehydration, anaemia, hypoglycaemia) appear to be the main causes of the high mortality that are potentially easy to manage. Due to the HIV pandemic, there has been low supply of blood and blood products for transfusion.

For populations in which the proportion of infants with LBW exceeds $10 \%$, SGA represents the majority of these infants(23). Kenya's LBW rate was reported at $12.8 \%$ in 1982 by $\mathrm{WHO}(17)$. With improved socio economic status, the contribution of SGA to the rate of LBW becomes less prominent. In view of the deteriorating socio economic situation in Kenya, the finding of an SGA rate of $11.6 \%$ may be an underestimate. This finding was based on maternal menstrual history which in a country such as Kenya is often inaccurate(23). In contrast Kasirye's and Dawodu's estimates may be more accurate at $19.9 \%$ and $55 \%$ respectively $(7,25)$. 
Compared to other morbidity events, mortality associated with SGA in our study was relatively lower than the overall rate. Some studies have shown that at comparable birth weights, infants who are SGA have lower neonatal and post neonatal mortality $(23,26)$. Our study findings are in agreement with these findings.

In contrast, Piper in Texas USA found that SGA preterm infants had significantly higher morbidity and mortality compared to appropriately grown preterm infants(27). If our SGA rate is an under estimate and Piper's findings are relevant to our situation, then the high mortality rate we are reporting is expected.

Confirmed sepsis as a morbidity event was associated with better survival. This is probably because with confirmation of septicaemia, the antibiotic sensitivity report offered guidance on the most suitable antibiotic hence the infant was better placed to survive. The organisms isolated were klebsiella, citrobacter, enterobacter, coagulase negative staphylococci and enterococci respectively. These are organisms that have previously been shown to cause neonatal sepsis in Kenya and other developing countries(3,19,22,28-30). Nathoo and co-workers in Zimbabwe found klebsiella sepsis as the leading cause of mortality especially in LBW and premature infants(31). Only one case of lancefield group B-Beta haemolytic streptococci was found. This organism while being responsible for most neonatal sepsis in developed countries is not prominent in developing countries $(3,29,32,33)$. No case of culture proven meningitis was identified in this study. The diagnosis of neonatal meningitis has remained elusive at the $\mathrm{KNH}$ not only because of the low rates of investigations (lumbar puncture), but also low yield on cerebrospinal fluid culture $(29,32)$. The reasons for this are yet to be identified.

Antibiotic use is still empirical in the unit as shown by the fact that more than half of the decisions to change antibiotics are not guided by laboratory sensitivity reports. Eighty seven percent of LBW infants admitted were on antibiotics yet only $37 \%$ had a diagnosis of suspected sepsis. Stoll et al acknowledges the increased use of antibiotics in very low birth weight infants in centers with better laboratory support(34). Very low birth weight infants formed $50 \%$ of the study population of this report and died before investigation or before the results were received in the unit, this may have contributed to the high rate of antibiotic use that was found.

Theophylline in the form of aminophylline is routinely given rectally to prevent apnoe in the LBW infants admitted to the unit. Presently KNH does not have facilities to monitor the serum levels of the drug despite its narrow therapeutic range. It is therefore not known whether the formulation and route of administration are achieving the intended purpose given the high rate of apnoea that was found.

Apart from intravenous dextrose as a 10 or $5 \%$ solution, nutritional support for the ill infant unable to tolerate enteral feeding is totally lacking. Partial parenteral nutrition that has been practiced in the unit before was not used in the LBW infants. This together with dehydration impacted negatively on LBW survival. While it is commendable that the main nutrient utilised is breast milk, it is only beneficial to those that can tolerate enteral feeding.

The overall finding of this study is a deteriorating mortality rate for LBW infants with increased morbidity over time. It has previously been shown in Nigeria that instituting selective measures can improve survival of neonates in a resource poor unit, although the improvement in this case was mainly realized for the larger babies(5). From Thailand, Ratrisawadi et al. also showed that effective neonatal care can have positive impact on morbidity and mortality of neonates across the birth weight categories(35). If a neonatal intensive care unit (NICU) is to be established in $\mathrm{KNH}$ in future, this should be on a background of prevailing optimal care based on the already existing facilities. It is only on this background that a NICU will provide maximum benefit(36).

Study Limitation: This was a retrospective study hence the researcher was not in control of the process. Poor record keeping may have an impact on the results obtained. However the overall results confirm otherwise anecdotal observation by the staff that the mortality appears to have increased over time.

Being retrospective may be an advantage in the sense that it assesses the actual practices which have not been altered as would happen in a prospective study situation where the research situation leads somewhat to improved care.

Conclusion: The mortality of LBW infants at $\mathrm{KNH}$ has deteriorated between 1978 and 2000 rising from about 270/1,000 admissions in 1978 to 574/ 1,000 admissions in 2000. This poor outcome is not only attributed to increased patient numbers (overcrowding) and understaffing but appears to be a direct result of inadequate care. The contribution of poor obstetric care (bad babies) could not be determined from this study.

Recommendations: It is urgent and necessary that written protocols for management of various common morbidities be formulated and strictly followed. To improve on the rates of respiratory failure resulting from respiratory distress, hypothermia, infection and poor nutritional support, the Kangaroo mother care method and a simple method to administer continous positive airways pressure in the absence of neonatal ventilators should be tried. Continous infection surveillance to determine changing patterns of infection and antibiotic sensitivity is required so as to have an informed (albeit empirical) choice of antibiotics. 


\section{REFERENCES}

1. Lubchenco, L.O, Searls, D.T. and Brazie, J.V. Neonatal mortality rate: Relationship to birth weight and gestational age. J. Pediatrics. 1972: 81:814-822.

2. Mcintire, D.D., Bloom, S.L., Casey, B.M. et al. Birth weight in relation to morbidity and mortality among new born infants. New Eng. J. Med. 1999; 340:1234 -1238

3. Stoll, B.J. The global impact of neonatal infections. Clin. Perinat. 1997; 24:1-21.

4. Were, F.N., Mukhwana, B.O. and Musoke, R.N. Neonatal survival of infants less than 2000 grams, born at Kenyatta National Hospital. East Afr. Med. J. 2002; 79:77-79.

5. Adekunle, H.D. and Effiong, C.E. Neonatal mortality: effects of selective pediatric interventions. Paediatrics. 1985; 75:51-57.

6. Meme J.S. A prospective study of neonatal death in Nairobi Kenya. East Afr. Med. J. 1978; 55:262-267.

7. Kasirye - Bainda, and Musoke R.N. Neonatal morbidity and mortality at Kenyatta National Hospital, new born unit. East Afr. Med. J. 1992; 69:360-365.

8. David, R.J. and Siegel, E. Decline in neonatal mortality; 1968 to 1977: better babies or better care? Pediatrics. 1983; 71:531-540.

9. Wasunna, A. and Mohammed, K. Morbidity and outcome of LBW babies of adolescent mothers at KNH-Nrb. East Afr. Med. J. 2002; 79:339-341

10. Wasunna, A. and Mohamed, K. LBW babies: Sociodemographic and obstetric characteristics of adolescent mothers at KNH-Nrb. East Afr. Med . J. 2002; 79:343-346.

11. Sarkar, C.S., Giri, A.K. and Sarkar, B. Outcome of teenage pregnancy and labour: a retrospective study. J. Indian Med. Assoc. 1991; 89:197-199.

12. Reichman, N.E. and Pagnini, D.L. Maternal age and birth outcomes. New Jersey Family planning perspectives. 1997; 29:268-272.

13. Simiyu, D.E. Morbidity and mortality of low birth weight infants in relation to maternal age at Kenyatta National Hospital. J. Obstet Gynecol Eastern Central Africa 2004; 17:25-33.

14. Keppel, K.G, Pearcy, J.Y. and Wagener, D.K. Trends in racial and ethnic specific rates for the health status indicators. United states, 1990-1998. Healthy people 2000 statistical notes/ national center for health statistics 2002; 23:1-16.

15. Zuckerman, B., Alpert, J.J., Dooling, E., et al. Neonatal outcome: is adolescent pregnancy a risk factor. Paediatrics. 1993; 71:489-493.

16. Elster, A.B. The effect of maternal age, parity and prenatal care on perinatal outcome in adolescent mothers. Amer. J. Obstet. Gynecol. 1984; 149:845-847.

17. Kramer, M.S. Determinants of low birth weight: methodological assessment and meta analysis. Bull World. Hlth. Org. 1987; 65:563-737.

18. Beeran, M., Solarin, K., Young, M. and Adelin, M. Morbidity and mortality of infants born before arrival at the hospital. Clin. Pediat. 1995; 34:313-316.

19. Lusweti, B.N. Antibiotic treatment in preterm babies born before arrival (BBA) at Kenyatta National Hospital. MMed Thesis 2001, University of Nairobi.
20. Jacob, J., Hulman, S. Davis, R.F. and Pfenninger, J. Racial differences in newborn intensive care morbidity in Alaska. Alaska Med. 2001; 43:32-37.

21. Melchor, J.C., Aranguren, G., Lopez, J.A. et al. Perinatal outcome of very low birth weight infants by mode of delivey. Int. J. Gynecol. Obstet. 1992; 38:181-187.

22. Admission and mortality data for year 2000 Kenyatta National Hospital records department.

23. McCormick, M.C. The contribution of low birth weight to infant mortality and childhood morbidity. New Eng. J. Med. 1985; 312:82-90.

24. Worku, B. The low birth weight infant in the Ethioswedish childrens' hospital, Addis Ababa. Ethiopian Med. J. 1999; 37:111-119.

25. Dawodu A.H. and Effiong C.E. Neonatal morbidity and mortality among Nigerian infants in a special care unit. East Afr. Med. J. 1983; 60:39.

26. Rivera - Rueda, M.A., Ramirez - Valdivia, J.M. Liz, Cedillo, R.E. et al. The perinatal aspects of surviving low birth weight neonates (Spanish- English abstract). Boletin medicio del hospital infantile de Mexico. 1992; 49:481-486.

27. Piper, J.M, .Xenakis, E.M., Mc Farland, M. et al. Do growth retarded premature infants have different rates of mortality than appropriately grown premature infants? Obstet. Gynaecol. 1996; 87:169-174.

28. Musoke, R.N. and Malenga, G.J. Bacterial infections in neonates at the Kenyatta National Hospital Nursery. A prospective study. East Afr. Med. J. 1984; 61:909-917.

29. Simiyu, D.E. Neonatal Septicemia in the General Paediatric wards at Kenyatta National Hospital. Medicom (in print).

30. H. J.J. Late onset infection in very low birth weight infants in Malaysian level 3. Neonatal nurseries. Malaysia very low birth weight study group. Pediatr. Infect. Dis. J. 2001;2:557-560.

31. Nathoo, K.J., Mason, P.R., Gwanzura, L. et al. Severe Klebsiella infection as a cause of mortality in neonates in Harare, Zimbabwe: evidence from postmortem blood cultures. Pediatr. Infect. Dis. J. 1983; 2:840-844.

32. Laving A., Neonatal bacterial meningitis at the new born unit of Kenyatta National Hospital. MMed Thesis 1997, University of Nairobi.

33. Onyango, F.E., Ndinya - Achola, J. et al. Lancefield group B-Beta Haemolytic streptococcal infections in the newborn at Kenyatta National Hospital. East Afr. Med. J. 1984; 61:376-381.

34. Stoll, B.J., Gordon, T., Korones, S.B. et al. Early onset sepsis in very low birth weight neonates: a report from the National Institute of Child Health and human development neonatal Research network. J. Pediatrics. 1996; 129:72-80.

35. Ratrisawadi, V., Aorpaopan, S. et al. Perinatal - neonatal and weight specific neonatal mortality in Thailand in 1996 and comparison with 1976 and 1986: a hospital based study. J. Med. Assoc. Thai. 1998; 506-511.

36. Blackman, J.A. Neonatal intensive care; is it worth it? Developmental sequelae of very low birth weight. Ped. Clin. N. Amer. 1991; 38:1497-1511. 